\title{
miR-485-5p inhibits bladder cancer metastasis by targeting HMGA2
}

\author{
ZHIJUN CHEN, QINGWEN LI, SHENG WANG and JIAJUN ZHANG
}

Department of Urinary Surgery, The First Affiliated Hospital of Bengbu Medical College, Bengbu, Anhui 233003, P.R. China

Received March 25, 2015; Accepted July 20, 2015

DOI: $10.3892 / \mathrm{ijmm} .2015 .2302$

\begin{abstract}
MicroRNA (miRNA or miR)-485 is a functional miRNA which has received much attention in recent years. However, little is known about the expression of miR-485 or the role it plays in bladder cancer [namely in metastasis and epithelial-mesenchymal transition (EMT)]. Thus, in the present study, we aimed to detect the expression of miR-485 in human bladder cancer tissues and bladder cancer cell lines, and to examine the effects of miR-485-5p on bladder cancer cell metastasis and EMT. We found that the expression of miR-485-5p was downregulated in the human bladder cancer tissues and different bladder cancer cell lines compared with the normal tissues and cell lines, as demonstrated by reverse transcription-quantitative polymerase chain reaction (RT-qPCR). We enforced the expression of miR-485-5p in T24 cells and inhibited the expression of miR-485-5p in SW780 cells by transfection with miR-485-5p mimic or miR-485-5p inhibitor, respectively. The ectopic expression of miR-485-5p was shown to inhibit cell metastasis and EMT, whereas the inhibition of miR-485-5p expression promoted cell metastasis and EMT, as shown by Transwell-Matrigel assay, cell adhesion assay and western blot analysis. Furthermore, a luciferase reporter assay revealed that high mobility group AT-hook 2 (HMGA2) was a direct target of miR-485-5p and that the overexpression of HMGA2 reversed the effects of miR-485-5p on cell metastasis and EMT. In conclusion and to the very best of our knowledge, the present study, for the first time, identified miR-485-5p as a suppressive miRNA in human bladder cancer, and demonstrated that miR-485-5p inhibits cell metastasis and EMT at least partly through the suppression of HMGA2 expression.
\end{abstract}

Correspondence to: Professor Qingwen Li, Department of Urinary Surgery, The First Affiliated Hospital of Bengbu Medical College, 287 Changhuai Road, Bengbu, Anhui 233003, P.R. China

E-mail: bblqw537@126.com

Key words: miR-485-5p, high mobility group AT-hook 2, bladder cancer, metastasis, epithelial-mesenchymal transition

\section{Introduction}

Bladder cancer is the most frequent urological malignancy in China and the second most common urological malignancy in the USA (1). Approximately 386,300 patients were diagnosed with bladder cancer in 2008, and 150,200 deaths were caused by this disease worldwide in the same year (2). The median survival of patients with bladder cancer is 15 months, and the 5 -year survival rate is only $15 \%$ (3). Therefore, it is critical to understand the molecular mechanisms involved in the development and progression of bladder cancer.

MicroRNAs (miRNAs or miRs) are a class of small non-coding RNA molecules, approximately $\sim 22$ nucleotides in length. They regulate target mRNAs at the post-transcriptional level and play crucial roles in the regulation of diverse physiological and pathological processes $(4,5)$. A number of miRNAs have been found to be linked with various types of cancer $(6,7)$. miR-485 is a functional miRNA which has received much attention in recent years. miR-485 acts as an important regulator of neural development and plasticity of the hippocampus (8). Recently, it has been demonstrated that miR-485 is associated with certain malignant tumors, including hepatocellular carcinomas, breast cancer and tumors of the central nervous system (9-12). However, little is known about the expression of miR-485 or its role in bladder cancer.

In the present study, we examined the expression of miR-485 in human bladder cancer tissues and bladder cancer cell lines, and examined the effects of miR-485-5p on cell metastasis and epithelial-mesenchymal transition (EMT). In addition, we explored the underlying mechanisms of the functions of miR-485 in bladder cancer cells and identified a novel direct target of miR-485-5p.

\section{Materials and methods}

Tumor samples. The bladder cancer tissue and the matched normal tissue specimens were collected from 15 patients diagnosed with bladder cancer who had undergone surgery, as the primary therapy, in The First Affiliated Hospital of Bengbu Medical College (Bengbu, China). None of the patients had received chemotherapy or radiotherapy prior to surgery. Prior to their participation in our study, all the patients provided written informed consent in compliance with the code of ethics of the Declaration of Helsinki. This study was approved by the Ethics Committee of The First Affiliated Hospital of Bengbu 
Medical College. The specimens were frozen in liquid nitrogen immediately after surgery.

Cell culture and transfection. Human bladder cancer cell lines (SW780, T24, HT1376 and HT5637) and human bladder epithelial cell lines HU609 and HEK293 cells (all from the American Type Culture Collection, Manassas, VA, USA) were cultured in Dulbecco's modified Eagle's medium (DMEM; Gibco-BRL, Grand Island, NY, USA) containing $10 \%$ newborn calf serum (Gibco-BRL) at $37^{\circ} \mathrm{C}$ with $5 \% \mathrm{CO}_{2}$. Prior to transfection, the cells were seeded in 6-well plates at $5 \times 10^{4}$ cells/well and allowed to grow for $24 \mathrm{~h}$. The miR-485-5p mimic (Bioneer, Shanghai, China), miR-485-5p inhibitor (Bioneer), miR control, the pcDNA3.1 (Zhonghong Boyuan Biological Technology Co., Ltd., Shenzhen, China) and high mobility group AT-hook 2 (HMGA2)-pcDNA3.1 (Zhonghong Boyuan Biological Technology Co., Ltd.) plasmids were transfected into the cells using a Lipofectamine ${ }^{\mathrm{TM}} 2000$ transfection kit (Invitrogen Life Technologies, Carlsbad, CA, USA) according to the manufacturer's instructions. Briefly, $2 \mu \mathrm{M}$ miR-485-5p mimic or inhibitor or plasmids were mixed with Lipofectamine 2000 to form lipid-DNA complexes. The cells were cultured in serum-free medium with the lipid-DNA complexes for $6 \mathrm{~h}$, and the medium was then replaced with fresh DMEM and the cells were maintained in the cultures for $24 \mathrm{~h}$.

Luciferase reporter assay. We used microRNA.org (http:// www.microrna.org/) and miRDB (http://mirdb.org/) to find the predicted target of miR-485-5p. Then, the HMGA2 wild-type and mutant $3^{\prime}$ untranslated region (UTR) were amplified and cloned into a psiCHECK-2 vector (Promega Corp., Madison, WI, USA). The cells were transfected with $100 \mathrm{ng}$ of the psiCHECK-2 vector and $100 \mathrm{nM}$ of the miR-485-5p mimic or miR control. After $24 \mathrm{~h}$, the cells were lysed and assayed using the Dual-Luciferase reporter assay system (Promega Corp.) according to the manufacturer's instructions.

Cell invasion assay. Cell invasion was examined using Matrigel (BD Biosciences, San Jose, CA, USA)-coated Transwell chambers (Corning Inc., Corning, NY, USA). The cells seeded at a density of $5 \times 10^{4}$ cells $/ \mathrm{ml}$ in serum-free medium were placed in the 1:8 diluted Matrigel-coated upper chamber. The lower chamber was filled with $1 \mathrm{ml}$ complete medium. Following incubation at $37^{\circ} \mathrm{C}$ for $24 \mathrm{~h}$, the cells that had invaded the bottom of the membrane were fixed in $95 \%$ ethanol for $20 \mathrm{~min}$ and then stained with hematoxylin. The cells on the surface of the membrane were removed using a cotton swab. The cells which had invaded were counted in 5 randomly selected microscopic fields.

Cell adhesion assay. The 96-well plates were coated with fibronectin (Sigma-Aldrich, St. Louis, MO, USA) and blocked with $1 \%$ bovine serum albumin (BSA; Sigma-Aldrich) in phosphate-buffered saline (PBS) at $37^{\circ} \mathrm{C}$ for $2 \mathrm{~h}$. The cells were suspended in serum-free medium and then seeded in the 96-well plates. Following incubation at $37^{\circ} \mathrm{C}$ for $2 \mathrm{~h}$, the non-adherent cells were removed by washing with PBS. The adherent cells were fixed with $4 \%$ paraformaldehyde and stained with $0.5 \%$ crystal violet.
$R N A$ extraction and reverse transcription-quantitative polymerase chain reaction $(R T-q P C R)$. Total RNA was extracted using an RNeasy/miRNeasy Mini kit (Qiagen, Limburg, The Netherlands) according to the manufacturer's instructions. A reverse transcriptase kit (Fermentas, Vilnius, Lithuania) was used to synthesize the cDNA. Quantitative PCR (qPCR) was carried out using SYBR-Green PCR Master Mix (Applied Biosystems, Foster City, CA, USA) on an ABI 7500 System (Applied Biosystems). The expression of miR-485-3p and miR-485-5p was examined and normalized to the internal control (GAPDH), and relative induction was calculated using the $2^{-\Delta \Delta \mathrm{Ct}}$ method.

Western blot analysis. Whole cell extracts were isolated using a Total Protein Extraction kit (Applygen Technologies, Inc., Beijing, China) according to the manufacturer's instructions. Proteins $(40 \mu \mathrm{g})$ were resolved by sodium dodecyl sulfate polyacrylamide gel electrophoresis (SDS-PAGE) and transferred onto a polyvinylidene fluoride membrane (Millipore, Billerica, MA, USA). The membrane was blocked with $5 \%$ BSA and probed with the primary antibodies [rabbit polyclonal to HMGA2 (ab97276), mouse monoclonal to E-cadherin (ab45990), rabbit polyclonal to vimentin (ab45939), mouse monoclonal to N-cadherin (ab98952) all from Abcam, Cambridge,MA,USA; mouse monoclonal to GAPDH (A01020), Amyjet Scientific, Inc., Wuhan, China]. The GAPDH antibody was used as an internal control. Antibody binding was detected with horseradish peroxidase (HRP)-linked secondary antibody [goat anti-mouse IgG (sc-2005), goat anti-rabbit IgG (sc-2004), Santa Cruz Biotechnology, Inc., Dallas, TX, USA] and the chemiluminescence (ECL western blotting kit; Pierce Biotechnology, Inc., Rockford, IL, USA).

Statistical analysis. SPSS 19.0 software (IBM, Armonk, NY, USA) was used to perform the statistical analysis. Data are expressed as the means \pm SD. The student's t-test was used to evaluate the differences between 2 groups. A P-value $<0.05$ was considered to indicate a statistically significant difference.

\section{Results}

Expression of miR-485 and HMGA2 in human bladder cancer cell lines. To detect the expression of miR-485-3p, miR-485-5p and HMGA 2 in human bladder cancer cell lines, we used 4 cell lines, SW780, T24, HT1376 and HT5637 from different grades of bladder cancer, and the human bladder epithelial cell line, HU609, was used as the normal control. The expression of miR-485-5p was highest in the HU609 cells, relatively lower in the SW780, HT1376 and HT5637 cells, and it was lowest in the poorly differentiated cell line, T24 (Fig. 1A). On the contrary, HMGA2 protein expression was lowest in the HU609 cells, a moderate expression was noted in the SW780, HT1376 and HT5637 cells, and a high expression was noted in the T24 cells (Fig. 1B). However, the expression level of miR-485-3p in the 4 bladder cancer cell lines did not differ significantly from that in the HU609 cells (Fig. 1A).

Expression of miR-485 and HMGA2 in human bladder cancer tissues. The expression levels of miR-485-3p, miR-485-5p and HMGA2 were detected in the human bladder cancer 

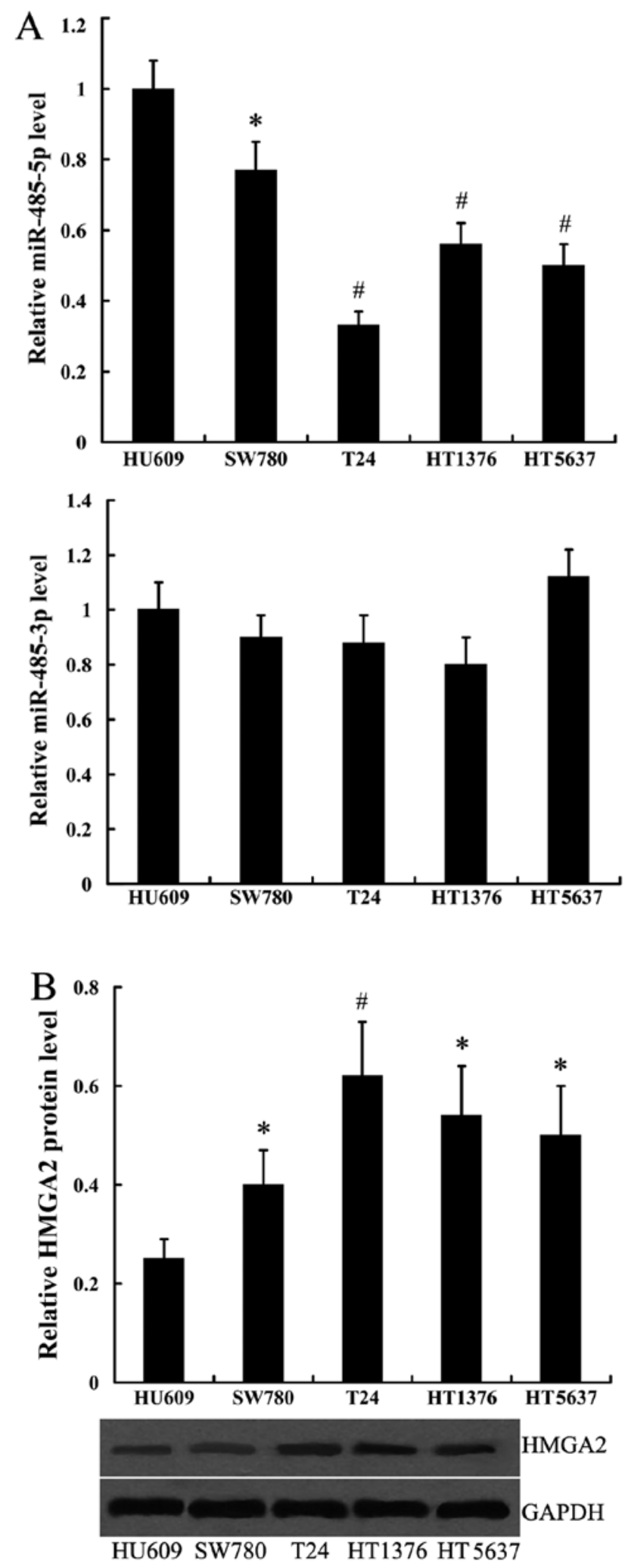

Figure 1.Expression of miR-485 and high mobility group AT-hook 2 (HMGA2) in human bladder cancer cell lines. (A) Relative mRNA levels of miR-485-5p and miR-485-3p; (B) relative protein levels of HMGA2 in the human bladder epithelial cell line, HU609, and 4 human bladder cancer cell lines. ${ }^{*} \mathrm{P}<0.05$ and ${ }^{\sharp} \mathrm{P}<0.01$ compared with HU609 cells.

tissues. Consistent with the results obtained from the human bladder cancer cell lines, the expression level of miR-485-5p was significantly decreased, whereas HMGA2 protein expres-
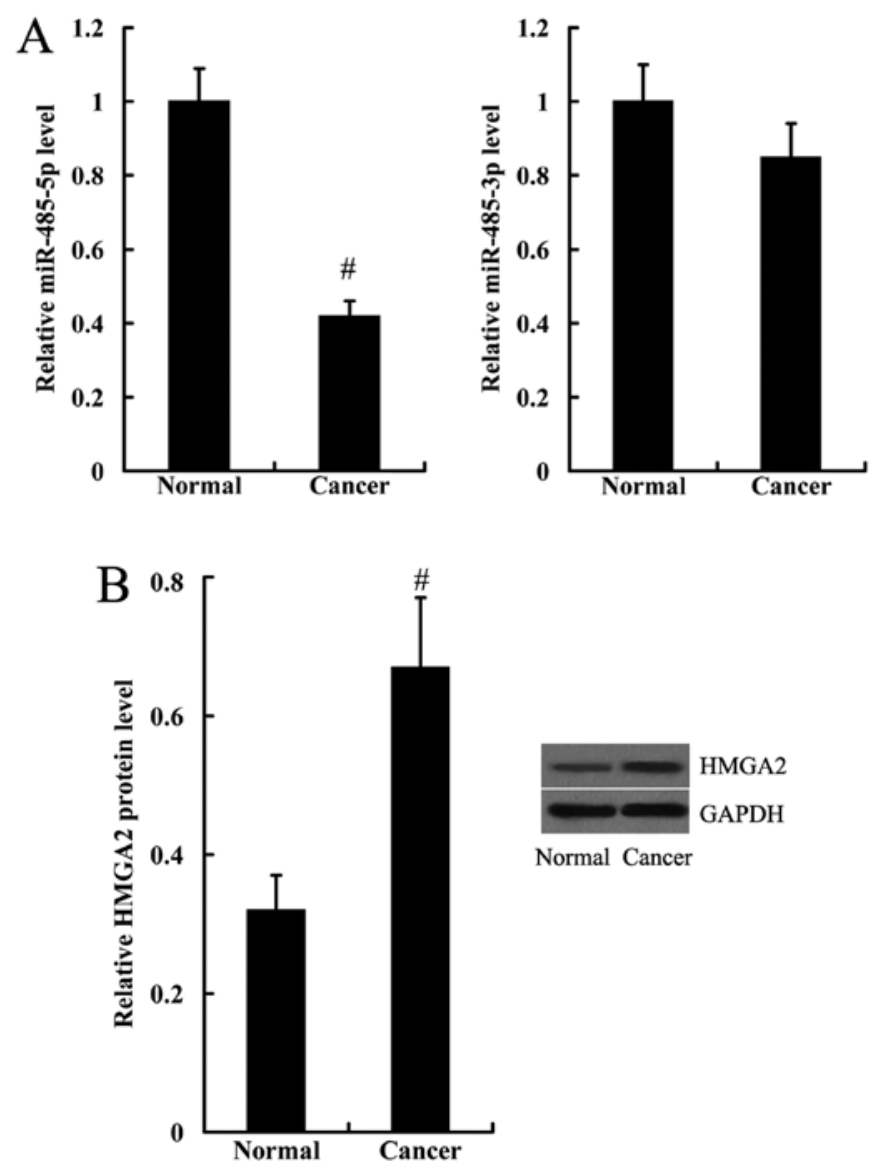

Figure 2. Expression of miR-485 and high mobility group AT-hook 2 (HMGA2) in human bladder cancer tissues. (A) Relative mRNA level of miR-485-5p and miR-485-3p; (B) relative protein level of HMGA2 in human bladder cancer tissues and matched normal tissues. ${ }^{~} \mathrm{P}<0.01$ compared with the normal tissues.

sion was significantly increased in the bladder cancer tissues, compared with the normal tissues (Fig. 2). The expression levels of miR-485-3p in the human bladder cancer tissues and normal tissues did not differ significantly (Fig. 2).

Effect of miR-485-5p on cell invasion and adhesion. To explore the effects of miR-485-5p on cell invasion and adhesion, T24 cells (which exhibited the lowest levels of miR-485-5p expression of the 4 bladder cancer cell lines examined) were transfected with the miR-485-5p mimic to induce the overexpression of miR-485-5p. We suppressed the expression of miR-485-5p in the SW780 cells, which exhibited a high miR-485-5p expression, by transfection with the miR-485-5p inhibitor. As demonstrated in Fig. 3A, transfection with the miR-485-5p mimic significantly increased the expression level of miR-485-5p, while transfection with the miR-485-5p inhibitor led to a significantly decrease in the expression of miR-485-5p. The results of Transwell-Matrigel assay revealed that the number of invaded cells was significantly decreased significantly when miR-485-5p was overexpressed. Moreover, the inhibition of miR-485-5p (by transfection with miR-485-5p inhibitor) promoted cell invasion compared with the cells transfected with the miR control (Fig. 3B). A cell adhesion assay revealed that adhesive ability decreased in the T24 cells transfected with the miR-485-5p mimic, but increased in the SW780 

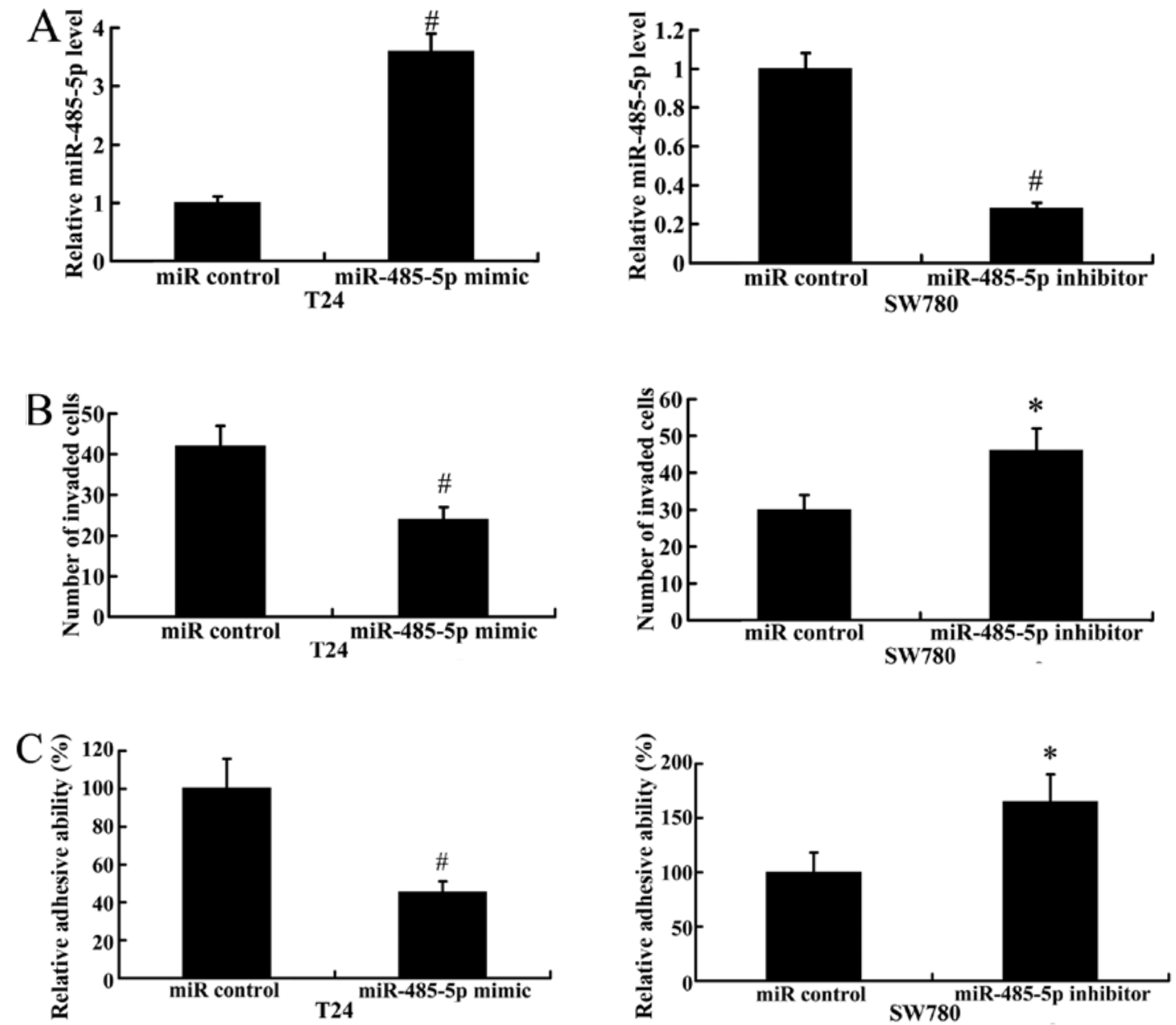

Figure 3. Effect of miR-485-5p on cell invasion and adhesion. (A) Relative mRNA levels of miR-485-5p in T24 and SW780 cells transfected with miR control or miR-485-5p mimic. (B) Number of invaded T24 and SW780 cells. (C) Adhesion ability of T24 and SW780 cells following transfection with the miR-485-5p mimic or miR-485-5p inhibitor. ${ }^{*} \mathrm{P}<0.05$ and ${ }^{\#} \mathrm{P}<0.01$ compared with the miR control-transfected cells.
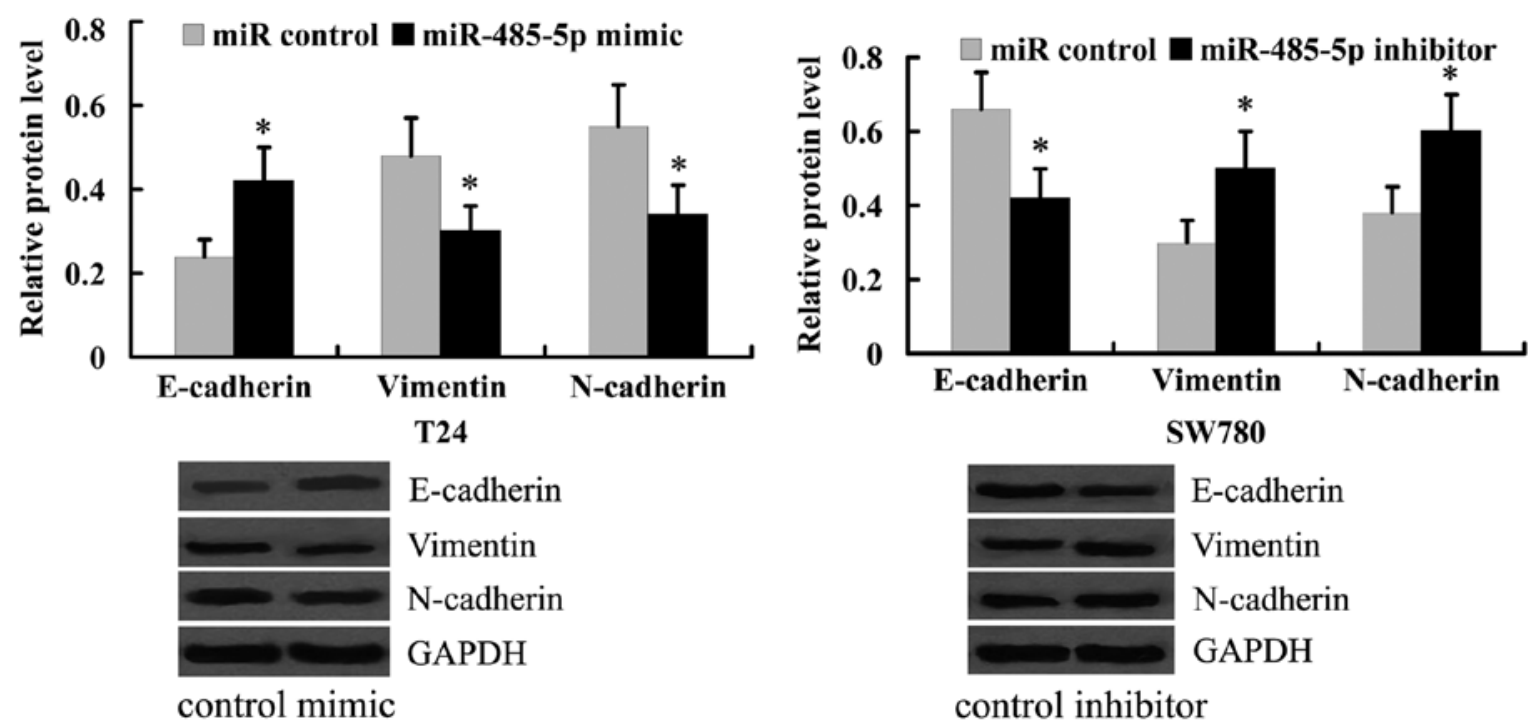

Figure 4. Expression of E-cadherin, vimentin and N-cadherin proteins in T24 and SW780 cells following transfection with the miR-485-5p mimic or miR-485-5p inhibitor. ${ }^{~} \mathrm{P}<0.05$ compared with the miR control-transfected cells.

cells transfected with the miR-485-5p-inhibitor, compared with the cells transfected with the miR control (Fig. 3C).
Effect of miR-485-5p on EMT. To examine the effect of miR-485-5p on EMT, the levels of the epithelial cell marker, 

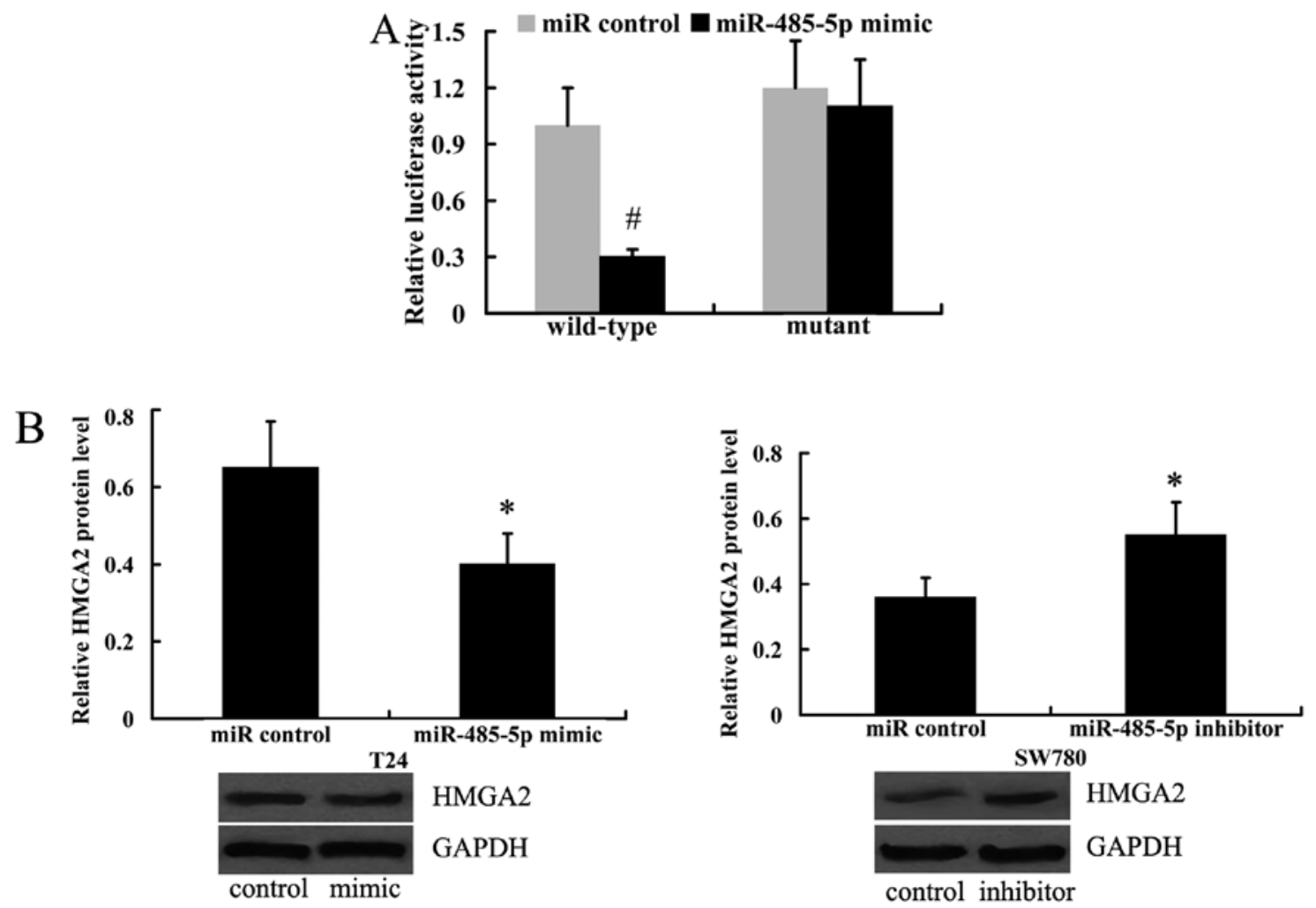

Figure 5. miR-485-5p directly targets the 3'UTR of high mobility group AT-hook 2 (HMGA2). (A) Relative luciferase activity in HEK293 cells following transfection with the HMGA2 wild-type 3'UTR or mutant 3'UTR vector and then the miR-485-5p mimic or miR control. (B) Relative protein level of HMGA2 in T24 and SW780 cells following transfection with the miR-485-5p mimic or miR-485-5p inhibitor. ${ }^{*} \mathrm{P}<0.05$ and ${ }^{\#} \mathrm{P}<0.01$ compared with the miR control-transfected cells.

E-cadherin, as well as the mesenchymal cell markers, vimentin and $\mathrm{N}$-cadherin, were detected in T24 and SW780 cells following transfection with the miR-485-5p mimic or miR-485-5p inhibitor. As shown in Fig. 4, transfection with the miR-485-5p mimic suppressed the protein expression of vimentin and $\mathrm{N}$-cadherin, whereas it promoted the expression of E-cadherin protein in the T24 cells. However, the inhibition of miR-485-5p with the miR-485-5p inhibitor in the SW780 cells induced EMT, evidenced by the increased protein expression of vimentin and $\mathrm{N}$-cadherin, and the decreased protein expression of E-cadherin.

miR-485-5p directly targets the 3'UTR of HMGA2. Using the prediction algorithm, microRNA.org (http://www.microrna. org/) and miRDB (http://mirdb.org/), HMGA2 was predicted to be a target of miR-485-5p. To determine the association between miR-485-5p and HMGA2, we performed a luciferase reporter assay to determine whether miR-485-5p directly targets the 3'UTR of HMGA2. The HEK293 cells were transfected with the HMGA2 wild-type 3'UTR or mutant 3'UTR vector and then with the miR-485-5p mimic or miR control. The results revealed that the luciferase activity of wild-type 3'UTR was significantly decreased by transfection with the miR-485-5p mimic. However, the luciferase activity of mutant 3'UTR was not altered substantially (Fig. 5A).

Subsequently, we investigated whether miR-485-5p regulates the expression of HsxMGA2 in the T24 and SW780 cells. As shown in Fig. 5B, miR-485-5p overexpression induced by transfection with miR-485-5p mimic resulted in a decrease in the HMGA2 protein level in the T24 cells. Moreover, the inhibition of miR-485-5p led to the upregulated protein expression of HMGA2 in the SW780 cells (Fig. 5B).

HMGA2 overexpression reverses the effects of miR-485-5p on cell metastasis and EMT. To investigate whether the effects of miR-485-5p on cell metastasis and ETM are related to HMGA2, the T24 cells overexpressing miR-485-5p were transfected with HMGA2-pcDNA3.1. As shown in Fig. 6A, the expression of HMGA2 was suppressed by the miR-485-5p mimic in T2 4 cells, and was significantly upregulated by transfection with HMGA2-pcDNA3.1. Moreover, we found that the inhibitory effects of miR-485-5p on cell invasion, adhesion and EMT were reversed by transfection with HMGA2-pcDNA3.1 (Fig. 6B-D).

\section{Discussion}

The differential expression profile of miRNAs has been widely reported between bladder cancer and normal cells (13). In the present study, we firstly demonstrated that the expression of miR-485-5p was downregulated in human bladder cancer tissues and various bladder cancer cell lines. These results suggest that miR-485-5p plays an important role in the development and progression of bladder cancer.

It has been demonstrated that miR-485-5p responds to hypoxic stress in soft tissue sarcoma cells (14). The expression of miR-485-5p was downregulated in Alzheimer's disease and malignant serous ovarian tumors, and correlated with FIGO grade in the latter (14), suggesting that miR-485-5p 

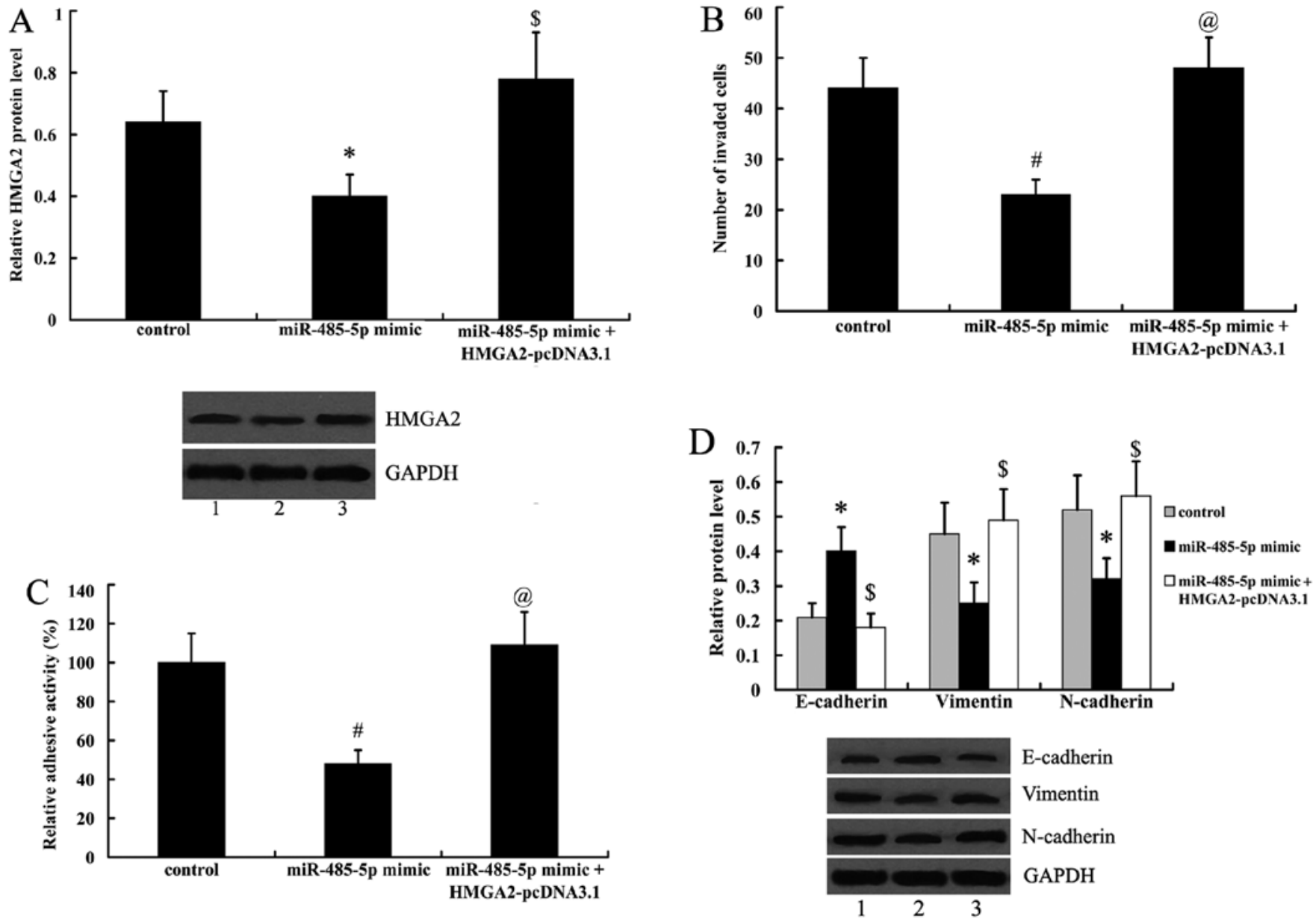

Figure 6. High mobility group AT-hook 2 (HMGA2) overexpression reverses the effect of miR-485-5p on cell metastasis and EMT. (A) Relative protein level of HMGA2 in T24 cells. (B) The number of invaded T24 cells. (C) Adhesion ability of T24 cells. (D) Expression of E-cadherin, vimentin and N-cadherin proteins in T24 cells following transfection with the miR-485-5p mimic and HMGA2-pcDNA3.1 plasmid. ${ }^{\mathrm{P}}<0.05$ and ${ }^{\#} \mathrm{P}<0.01$ compared with the miR control-transfected cells; ${ }^{\top} \mathrm{P}<0.05$ and ${ }^{\circledR} \mathrm{P}<0.01$ compared with the miR-485-5p mimic-transfected cells. Lane 1, control group; lane 2, miR-485-5p mimic-transfected group; lane 3, miR-485-5p mimic + HMGA2 pcDNA3.1-transfected group.

may be of potential importance as a diagnostic biomarker. Consistently, miR-485-3p is downregulated in metastatic cell lines as compared with normal prostatic epithelial cells (11). In addition, it has been found that miR-485 suppresses the proliferation and migration of breast carcinoma T47D cells (12). In the present study, we performed cell invasion and adhesion assays to investigate the effects of miR-485-5p on bladder cancer cell metastasis. It is well known that EMT is a well-coordinated process for metastasis $(15,16)$; therefore, the effects of miR-485-5p on EMT were also examined. The results demonstrated that miR-485-5p inhibited bladder cancer cell metastasis and EMT.

In the present study, we examined HMGA2 as a novel direct target of miR-485-5p. It has been demonstrated elsewhere that HMGA2, an architectural transcription factor,plays a role in certain cellular biological processes, such as cell proliferation, differentiation, cell transformation and cell cycle control (17-19). HMGA2 is expressed predominantly in various undifferentiated tissues during embryonic development, whereas it is usually not detectable in normal adult tissues (20-23). The heightened expression of HMGA2 in adult tissues is commonly associated with various human cancers (24-28), such as lung, breast and ovarian cancer. It has been reported that HMGA2 is upregulated in bladder cancer at both the transcriptional and translational level, and it has been noted that HMGA2 expression is a potential prognostic marker for both tumor recurrence and tumor progression (29). Moreover, a it has been previously demonstrated that the expression of HMGA2 is closely associated with EMT in bladder cancer (30). Consistent with previous reports, in the present study, we found that the protein expression of HMGA2 was upregulated in human bladder cancer tissues and bladder cancer cell lines. To examine the association between miR-485-5p and HMGA2, we searched for the potential target genes of miR-485-5p using the prediction algorithm, microRNA.org (http://www.microrna.org/) and miRDB (http:// mirdb.org/), and HMGA2 was predicted to be a target gene. Using a luciferase reporter assay and western blot analysis, we demonstrated that miR-485-5p suppressed HMGA2 expression by directly targeting the 3 'UTR of HMGA2. In addition, using the HMGA2 overexpression plasmid, HMGA2-pcDNA3.1, we found that HMGA2 reversed the effects of miR-485-5p on cell metastasis and EMT. These results suggest that HMGA2 plays an important role in mediating the effects of miR-485-5p. 
Taken together, to the best of our knowledge, the present study is the fist to identify miR-485-5p as a suppressive miRNA in human bladder cancer. In addition, we demonstrated that miR-485-5p exerts its effect at least partly through the suppression of HMGA2. Our study further expanded on the function of miR-485 and provided a novel potential target for the treatment of bladder cancer.

\section{References}

1. Zhu Z, Shen Z and Xu C: Inflammatory pathways as promising targets to increase chemotherapy response in bladder cancer. Mediators Inflamm 2012: 528690, 2012.

2. Jemal A, Bray F, Center MM, Ferlay J, Ward E and Forman D: Global cancer statistics. CA Cancer J Clin 61: 69-90, 2011.

3. von der Maase H, Sengelov L, Roberts JT, Ricci S, Dogliotti L, Oliver T, Moore MJ, Zimmermann A and Arning M: Long-term survival results of a randomized trial comparing gemcitabine plus cisplatin, with methotrexate, vinblastine, doxorubicin, plus cisplatin in patients with bladder cancer. J Clin Oncol 23 4602-4608, 2005.

4. Ambros V: The functions of animal microRNAs. Nature 431: 350-355, 2004.

5. Bartel DP: MicroRNAs: Genomics, biogenesis, mechanism, and function. Cell 116: 281-297, 2004.

6. Kusenda B, Mraz M, Mayer J and Pospisilova S: MicroRNA biogenesis, functionality and cancer relevance. Biomed Pap Med Fac Univ Palacky Olomouc Czech Repub 150: 205-215, 2006.

7. Mraz M, Pospisilova S, Malinova K, Slapak I and Mayer J: MicroRNAs in chronic lymphocytic leukemia pathogenesis and disease subtypes. Leuk Lymphoma 50: 506-509, 2009.

8. Cohen JE, Lee PR and Fields RD: Systematic identification of 3'-UTR regulatory elements in activity-dependent mRNA stability in hippocampal neurons. Philos Trans R Soc Lond B Biol Sci 369: 20130509, 2014.

9. Yang H, Cho ME, Li TW, Peng H, Ko KS, Mato JM and Lu SC: MicroRNAs regulate methionine adenosyltransferase 1A expression in hepatocellular carcinoma. J Clin Invest 123: 285-298, 2013.

10. Costa FF, Bischof JM, Vanin EF, Lulla RR, Wang M, Sredni ST, Rajaram V, Bonaldo MF, Wang D, Goldman S, et al: Identification of microRNAs as potential prognostic markers in ependymoma. PLoS One 6: e25114, 2011.

11. Formosa A, Markert EK, Lena AM, Italiano D, Finazzi-Agro' E, Levine AJ, Bernardini S, Garabadgiu AV, Melino G and Candi E: MicroRNAs, miR-154, miR-299-5p, miR-376a, miR-376c miR-377, miR-381, miR-487b, miR-485-3p, miR-495 and miR-654-3p, mapped to the $14 q 32.31$ locus, regulate proliferation, apoptosis, migration and invasion in metastatic prostate cancer cells. Oncogene 33: 5173-5182, 2014.

12. Anaya-Ruiz M, Bandala C and Perez-Santos JL: miR-485 acts as a tumor suppressor by inhibiting cell growth and migration in breast carcinoma T47D cells. Asian Pac J Cancer Prev 14: 3757-3760, 2013.

13. Catto JW, Alcaraz A, Bjartell AS, De Vere White R, Evans CP, Fussel S, Hamdy FC, Kallioniemi O, Mengual L, Schlomm T, and Visakorpi T: MicroRNA in prostate, bladder, and kidney cancer: a systematic review. Eur Urol 59: 671-681, 2011.

14. Gits CM, van Kuijk PF, de Rijck JC, Muskens N, Jonkers MB, van IJcken WF, Mathijssen RH, Verweij J, Sleijfer S and Wiemer EA: MicroRNA response to hypoxic stress in soft tissue sarcoma cells: microRNA mediated regulation of HIF3 $\alpha$. BMC Cancer 14: 429, 2014.
15. Thiery JP: Epithelial-mesenchymal transitions in tumour progression. Nat Rev Cancer 2: 442-454, 2002.

16. Brabletz T, Hlubek F, Spaderna S, Schmalhofer O, Hiendlmeyer E, Jung A and Kirchner T: Invasion and metastasis in colorectal cancer: epithelial-mesenchymal transition, mesenchymalepithelial transition, stem cells and beta-catenin. Cells Tissues Organs 179: 56-65, 2005.

17. Di Cello F, Hillion J, Hristov A, Wood LJ, Mukherjee M, Schuldenfrei A, Kowalski J, Bhattacharya R, Ashfaq R and Resar LM: HMGA2 participates in transformation in human lung cancer. Mol Cancer Res 6: 743-750, 2008.

18. Lanahan A, Williams JB, Sanders LK and Nathans D: Growth factor-induced delayed early response genes. Mol Cell Biol 12: 3919-3929, 1992

19. Narita M, Narita M, Krizhanovsky V, Nuñez S, Chicas A, Hearn SA, Myers MP and Lowe SW: A novel role for high-mobility group a proteins in cellular senescence and heterochromatin formation. Cell 126: 503-514, 2006.

20. Gattas GJ, Quade BJ, Nowak RA and Morton CC: HMGIC expression in human adult and fetal tissues and in uterine leiomyomata. Genes Chromosomes Cancer 25: 316-322, 1999.

21. Zhou X, Benson KF, Ashar HR and Chada K: Mutation responsible for the mouse pygmy phenotype in the developmentally regulated factor HMGI-C. Nature 376: 771-774, 1995.

22. Ashar HR, Chouinard RA Jr, Dokur M and Chada K: In vivo modulation of HMGA2 expression. Biochim Biophys Acta 1799: 55-61, 2010.

23. Pfannkuche K, Summer H, Li O, Hescheler J and Dröge P: The high mobility group protein HMGA2: A co-regulator of chromatin structure and pluripotency in stem cells? Stem Cell Rev 5: 224-230, 2009.

24. Piscuoglio S, Zlobec I, Pallante P, Sepe R, Esposito F, Zimmermann A, Diamantis I, Terracciano L, Fusco A and Karamitopoulou E: HMGA1 and HMGA2 protein expression correlates with advanced tumour grade and lymph node metastasis in pancreatic adenocarcinoma. Histopathology 60: 397-404, 2012.

25. Wu Y, Song Y and Liu H: Expression and its clinical significance of HMGA2 in the patients with non-small cell lung cancer. Zhongguo Fei Ai Za Zhi 11: 377-381, 2008 (In Chinese).

26. Langelotz C, Schmid P, Jakob C, Heider U, Wernecke KD, Possinger K and Sezer O: Expression of high-mobility-groupprotein HMGI-C mRNA in the peripheral blood is an independent poor prognostic indicator for survival in metastatic breast cancer. Br J Cancer 88: 1406-1410, 2003.

27. Malek A, Bakhidze E, Noske A, Sers C, Aigner A, Schäfer R and Tchernitsa O: HMGA2 gene is a promising target for ovarian cancer silencing therapy. Int J Cancer 123: 348-356, 2008.

28. Wang X, Liu X, Li AY, Chen L, Lai L, Lin HH, Hu S, Yao L, Peng J, Loera S, et al: Overexpression of HMGA2 promotes metastasis and impacts survival of colorectal cancers. Clin Cancer Res 17: 2570-2580, 2011.

29. Yang GL, Zhang LH, Bo JJ, Hou KL, Cai X, Chen YY, Li H, Liu DM and Huang YR: Overexpression of HMGA2 in bladder cancer and its association with clinicopathologic features and prognosis HMGA2 as a prognostic marker of bladder cancer. Eur J Surg Oncol 37: 265-271, 2011.

30. Ding X, Wang Y, Ma X, Guo H, Yan X, Chi Q, Li J, Hou Y and Wang C: Expression of HMGA2 in bladder cancer and its association with epithelial-to-mesenchymal transition. Cell Prolif 47: 146-151, 2014. 